\title{
Prognostic capacity of hyperdense middle cerebral artery sign in anterior circulation acute ischaemic stroke patients receiving reperfusion therapy: a systematic review and meta-analysis
}

\author{
Chenyu Shi ${ }^{1,3} \cdot$ Murray C. Killingsworth ${ }^{1,5,7,9} \cdot$ Sonu Menachem Maimonides Bhaskar ${ }^{1,2,4,5,6,8}$ (D)
}

Received: 6 April 2021 / Accepted: 31 May 2021 / Published online: 6 June 2021

๑) Belgian Neurological Society 2021

\begin{abstract}
Pre-intervention CT imaging-based biomarkers, such as hyperdense middle cerebral artery sign (HMCAS) may have a role in acute ischaemic stroke prognostication. However, the clinical utility of HMCAS in settings of reperfusion therapy and the level of prognostic association is still unclear. This systematic review and meta-analysis investigated the association of HMCAS sign with clinical outcomes and its prognostic capacity in acute ischaemic stroke patients treated with reperfusion therapy. Prospective and retrospective studies from the following databases were retrieved from EMBASE, MEDLINE and Cochrane. Association of HMCAS with functional outcome, symptomatic intracerebral haemorrhage (sICH) and mortality were investigated. The random effect model was used to calculate the risk ratio (RR). Subgroup analyses were performed for subgroups of patients receiving thrombolysis (tPA), mechanical thrombectomy (EVT) and/or combined therapy (tPA + EVT). HMCAS significantly increased the rate of poor functional outcome by 1.43 -fold in patients (RR 1.43; 95\% CI 1.30-1.57; $p<0.0001$ ) without any significant differences in sICH rates (RR 0.91; 95\% CI 0.68-1.23; $\mathrm{p}=0.546$ ) and mortality (RR $1.34 ; 95 \% \mathrm{CI} 0.72-2.51 ; p=354$ ) in patients with positive HMCAS as compared to negative HMCAS. In subgroup analyses, significant association between HMCAS and 90 days functional outcome was observed in patients receiving tPA (RR 1.53; $95 \%$ CI $1.40-1.67 ; p<0.0001$ ) or both therapies (RR 1.40; 95\% CI 1.08-1.80; $p=0.010$ ). This meta-analysis demonstrated that pre-treatment HMCAS increases risk of poor functional outcomes. However, its prognostic sensitivity and specificity in predicting long-term functional outcome, mortality and sICH after reperfusion therapy is poor.
\end{abstract}

Keywords HMCAS $\cdot$ Stroke $\cdot$ Endovascular therapy $\cdot$ Functional outcome $\cdot$ Symptomatic intracerebral haemorrhage $\cdot$ Mortality $\cdot$ Meta-analysis

Sonu Menachem Maimonides Bhaskar

Sonu.Bhaskar@reprogramglobal.org

Chenyu Shi

chenyu.shi1@ student.unsw.edu.au

Murray C. Killingsworth

Murray.Killingsworth@health.nsw.gov.au

1 Neurovascular Imaging Laboratory, Clinical Sciences Stream, Ingham Institute for Applied Medical Research, Sydney, Australia

2 South West Sydney Local Health District (SWSLHD), Sydney, Australia

3 South Western Sydney Clinical School, University of New South Wales (UNSW), Sydney, NSW, Australia

4 Stroke and Neurology Research Group, Ingham Institute for Applied Medical Research, Sydney, Australia
5 NSW Brain Clot Bank, NSW Health Statewide Biobank and NSW Health Pathology, Sydney, NSW, Australia

6 Thrombolysis and Endovascular WorkFLOw Network (TEFLON), Sydney, Australia

7 Correlative Microscopy Facility, Ingham Institute for Applied Medical Research, Sydney, Australia

8 Present Address: Department of Neurology and Neurophysiology, Liverpool Hospital, Clinical Sciences Building, Elizabeth St, Liverpool, NSW 2170, Australia

9 Department of Anatomical Pathology, NSW Health Pathology and Liverpool Hospital, Liverpool, NSW, Australia 


$\begin{array}{ll}\text { Abbreviations } \\ \text { HMCAS } & \text { Hyperdense middle cerebral artery } \\ \text { NCCT } & \text { Non-contrast computed tomography } \\ \text { AIS } & \text { Acute ischaemic stroke } \\ \text { EVT } & \text { Endovascular thrombectomy } \\ \text { tPA } & \text { Tissue-plasminogen activator } \\ \text { CTA } & \text { Computed tomography angiography } \\ \text { IV } & \text { Intravenous } \\ \text { CTP } & \text { Computed tomography perfusion } \\ \text { SICH } & \text { Symptomatic intracerebral haemorrhage } \\ \text { SITS-ISTR } & \text { Thrombolysis in Stroke International Stroke } \\ & \text { Thrombolysis Register } \\ \text { MCA } & \text { Middle cerebral artery } \\ \text { RR } & \text { Risk ratio }\end{array}$

\section{Introduction}

Non-contrast computed tomography (NCCT) remains the primary imaging modality in acute ischaemic stroke (AIS) $[1,2]$. Early recognition of AIS on imaging is important to decide the optimal therapeutic strategy [1, 2]. Hyperdense middle cerebral artery sign (HMCAS) is a focal and tubular hyper-density area often seen on NCCT in the middle cerebral artery (MCA) during the hyperacute phase [3, 4]. The prevalence of HMCAS is approximately $19-40 \%$ in acute MCA stroke patients [5, 6]. It has been shown to delineate the location of the occlusion and baseline stroke severity [3]. The wide availability of NCCT in comparison to advanced imaging modalities, such as computed tomography perfusion imaging (CTP) makes it easier to appreciate HMCAS in real-world settings $[1,7]$. The HMCAS has also been used to investigate the morphological characteristics of the clot as a surrogate imaging biomarker $[4,5,7]$.

The reperfusion strategies, using pharmacological or intravenous thrombolysis (IVT) and endovascular thrombectomy (EVT), is currently the mainstay of acute ischaemic stroke treatment [8]. Despite the emerging role of HMCAS, its prognostic role in clinical settings, in settings of reperfusion therapy, needs further consideration. Indeed, the clot composition (such as the degree of clot density, relative composition of red blood cells (RBCs) [9]) in addition to other factors, such as time to reperfusion [10], baseline stroke severity [11], collateral status [12, 13], etc., are associated with the efficacy of the EVT $[14,15]$. This study sought to investigate the association of HMCAS with clinical outcomes and its prognostic capacity in anterior circulation AIS patients receiving reperfusion therapy by performing a systematic review and meta-analysis.

\section{Methods}

\section{Literature search: identification and selection of studies}

Medline, Embase and Cochrane central register of controlled trials were used as the search engines. Articles were searched between January 2000 and August 2020. The search terms included: "hyperdense MCA sign" or "hyperdense artery sign" or "hyperdensity artery sign" or "HDMCA" or "HMCAS" in association with "stroke" or "anterior circulation" or "acute ischaemic stroke" and "Thrombectomy" or "Thrombolytic therapy" or "Tissue plasminogen activator" or "tPA" or "outcomes". Additional limits were applied to exclude studies that were not in the English language and those that didn't include human subjects. The full search term is available in the Supplementary Information (search strategy). The Preferred reporting items for systematic reviews and metaanalysis (PRISMA) checklist was adopted in this metaanalysis [16].

\section{Inclusion and exclusion criteria}

Studies were eligible if they met the following criteria: (1) patients were aged 18 years or above; (2) patients had been diagnosed with AIS, (3) patients received endovascular therapy and/or mechanical thrombectomy; (4) studies with good methodological design and (5) studies with sufficient sample size, determined to be $>20$ patients in each group. The exclusion criteria were: (1) animal studies; (2) duplicated publications; (3) full-text article not available; (4) systematic reviews, meta-analyses, letters and case reports or case series and (5) studies presented in the abstract form, with relevant data on HMCAS not available or no relevant post-reperfusion clinical outcome measured were excluded.

\section{Data extraction}

The title and abstracts were reviewed on Endnote first to rule out the articles mismatched to the eligibility criteria. The remaining articles were examined thoroughly to determine whether they should be included for the systematic review or meta-analysis according to the eligibility criteria. The screening was conducted independently by two authors. Disagreements were discussed and final decisions were reaches at by consensus. The data from each study/trial were extracted independently using a data extraction sheet to obtain the following information: (1) baseline demographics: author, country and year of publication; (2) study population: age of patients, sample 
size, characteristics of acute stroke patients, presence or absence of HMCAS; (3) type and time window of the treatments and (4) outcome measures: functional outcome, sICH and mortality at 90 days.

\section{Quality assessment of included studies}

The methodological quality of each study was assessed independently by two researchers using the Newcastle Ottawa scale [17]. The scale evaluates the study quality based on the following evaluation criteria: Selection, comparability and outcomes. The total score for each study ranged from 0 to 9 points and using the nine items. National Institute of Health (NIH) Quality Assessment Tool for observational cohort and cross-sectional studies was also used for the quality assessment of the included studies. The total score for each study ranged from 0 to 14 points [18]. The risk of bias in results due to funding was evaluated, independently from the quality assessment, through the declaration of funding sources and conflicts of interest using the previously reported scoring test [19]. A score of 1-2 was considered to indicate a moderate potential for bias. The absence of industry funding was not taken to signify an absence of bias, but the presence of industry funding or conflicts of interest was assumed to be an indicator of bias.

\section{Statistical analysis}

All statistical analyses were performed using STATA (Version 13.0, StataCorp LLC, College Station, Texas, USA). In this study, functional outcome, sICH and mortality were compared in patients with or without HMCAS. The Forest plots were generated to present the risk ratios (RR), 95\% confidence intervals (CI), percentage weight and the heterogeneity between studies included in the meta-analysis. $I^{2}$ statistics and $p$ values were used to assess the heterogeneity between the studies $(25 \%=$ low, $50 \%=$ medium, $75 \%=$ high). The random effects model was used across all subgroup analyses. Subgroup analysis for patients receiving tissue plasminogen activator (tPA), mechanical thrombectomy (EVT) or both were also performed. The baseline characteristics of patients' populations were synthesized from all included studies. Where applicable, median and interquartile ranges were converted to mean and standard deviation using the method described by Wan et al. 2012 [20]. A (Begg's) funnel plot was used to visually detect the presence of publication bias in the meta-analysis. Asymmetry on either side of the funnel plot is indicative of the presence of publication bias. Sensitivity and specificity analysis were also used in the detection of prognostic performance in meta-analysis. $p$ values $<0.05$ were considered statistically significant (Fig. 1).

\section{Results}

\section{Description of included studies}

The total number of patients included in this study was 12,329 , of which 7118 (57.7\%) were male and 2724 patients (22.1\%) were positive for HMCAS on pre-intervention NCCT. The mean age \pm SD (range) of all included studies was $68.4 \pm 15.6$ years. The clinical characteristics are shown in Table 1. Definitions of poor functional outcome and sICH are varied across studies. The results of methodological quality, risk of bias and funding bias assessment of included studies are provided in Supplementary Information (Supplementary Tables 1, 2 and 3). Effect size analyses for functional outcome, sICH and mortality is also presented in Supplementary Information (Supplementary Figs. 1, 2 and 3).

\section{Association of HMCAS with long-term functional outcome}

Overall, 12 studies were included in the final meta-analysis of association of HMCAS with the functional outcome at 90 days comprising of 11,894 patients [21-32]. A poor functional outcome was defined as a 90 -days modified Rankin score (mRS) of 3-6 in 8 studies [21, 23-28, 30] and 2-6 in 4 studies [22, 29, 31, 32]. HMCAS significantly increased the risk of poor functional outcome by 1.43 -fold (RR 1.43; 95\% CI 1.30-1.57; $p<0.0001$ ) (Fig. 2). Moderate heterogeneity was found between the studies $\left(I^{2}=55.1 \%\right.$, $p=0.011)$. No evidence of publication bias was observed by visual inspection of the funnel plot (Fig. 5). Significant association of HMCAS with functional outcome at 90 days was observed in patients receiving tPA (RR 1.53; 95\% CI $1.40-1.67 ; p<0.0001$ ) or both therapies (RR $1.40 ; 95 \% \mathrm{CI}$ $1.08-1.80 ; p=0.010$ ) (Fig. 2). No significant association of HMCAS with functional outcome at 90 days was observed in patients receiving EVT (RR 1.16; 95\% CI 0.99-1.37; $p=0.071)$. The sensitivity and specificity of HMCAS stratified by reperfusion therapy are provided in Table 2 .

\section{Association of HMCAS with sICH}

Ten studies consisting of 11,871 patients were included in the final meta-analysis of the association of HMCAS with sICH [22, 23, 25-30, 33, 34]. Most of the studies defined sICH according to SITS-MOST criteria. SITS-MOST criteria define $\mathrm{sICH}$ as local or remote $\mathrm{PH} 2$ (parenchymal haemorrhage) seen on $\mathrm{CT}$ at the $22-36 \mathrm{~h}$ combined with a neurological deterioration of 4 points on the baseline NIHSS or leading to death [23, 25, 28]. Other definitions of sICH used in these studies are described in Table 1. 


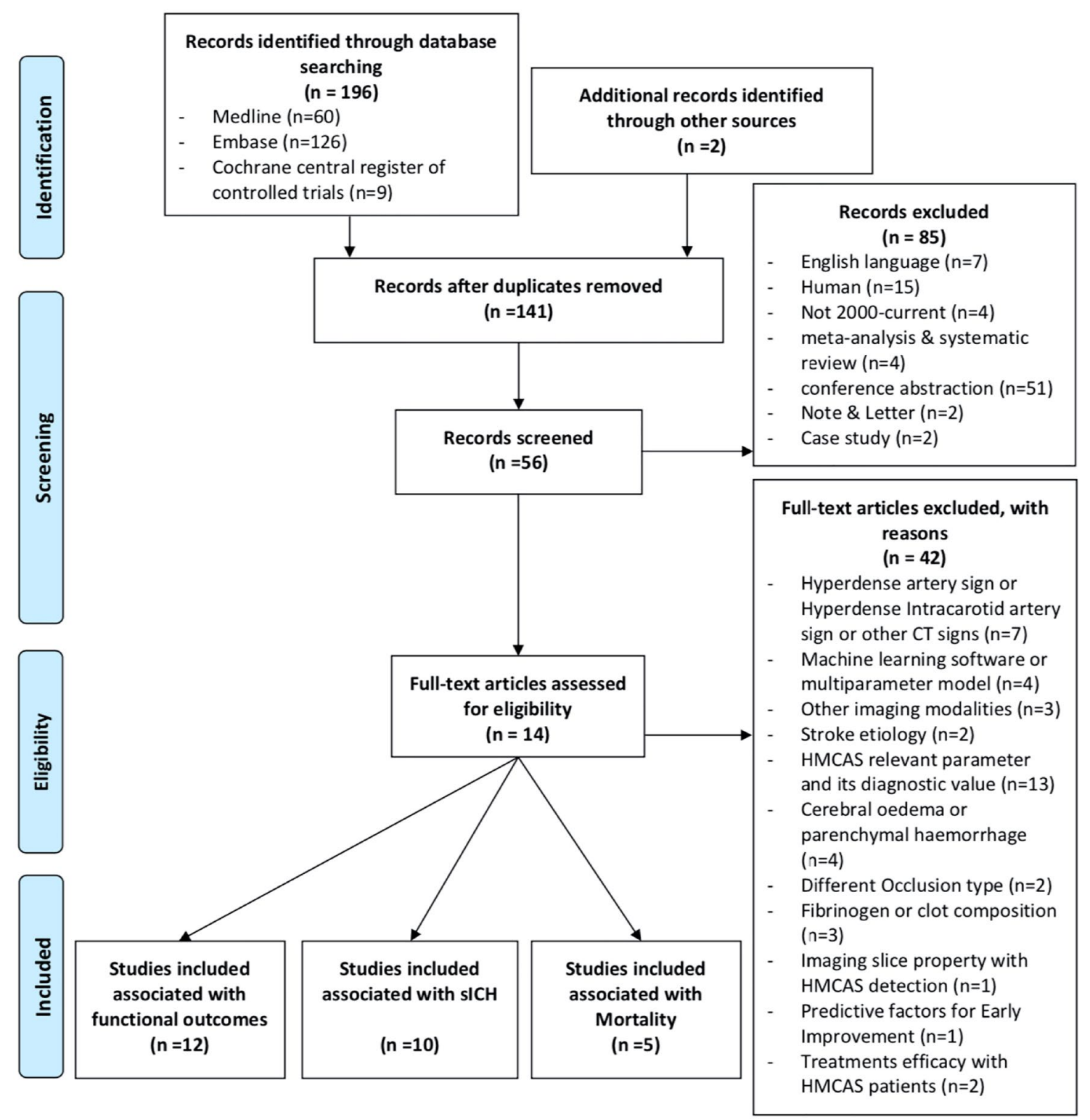

Fig. 1 The PRISMA flowchart showing the main characteristics of included studies

No significant association of HMCAS with sICH was observed (RR 0.91; 95\% CI 0.68-1.23; $p=0.546$; Fig. 3). Low heterogeneity was found between studies $\left(I^{2}=0.0 \%\right.$, $p=0.705)$. No evidence of publication bias was observed on the funnel plot (Fig. 5). For patients receiving tPA, the HMCAS was associated with decreased risk of sICH rates, though the association was not significant (RR 0.86; 95\% CI 0.64-1.16; $p=0.329$; Fig. 3). In EVT patients, HMCAS was associated with an increased risk of sICH, however it failed to reach statistical significance (RR $3.95 ; 95 \%$ CI $0.88-17.67$; $p=0.0 .072$; Fig. 3 ). The sensitivity and specificity are shown for tPA and all included studies in Table 2.

\section{Association of HMCAS with mortality}

Five studies were included in the final analysis of association of HMCAS with mortality which comprised of 10,922 patients [21, 25, 26, 28, 33] (Table 1). HMCAS was not significantly associated with increased risk of mortality in patients with positive HMCAS as compared to negative HMCAS (RR 1.34; 95\% CI 0.72-2.51; $p=0.354$ ) (Fig. 4). The heterogeneity $\left(I^{2}\right)$ was found to be high $(88.0 \%$, $p<0.001)$ across studies. There is no evidence of publication bias upon visual inspection of the funnel plot (Fig. 5). The association of HMCAS with mortality risk was not significant in patients who received thrombolysis (RR 1.26; 95\% CI 0.61-2.62; $p=0.531$ ) (Fig. 4). Meta-analysis on 


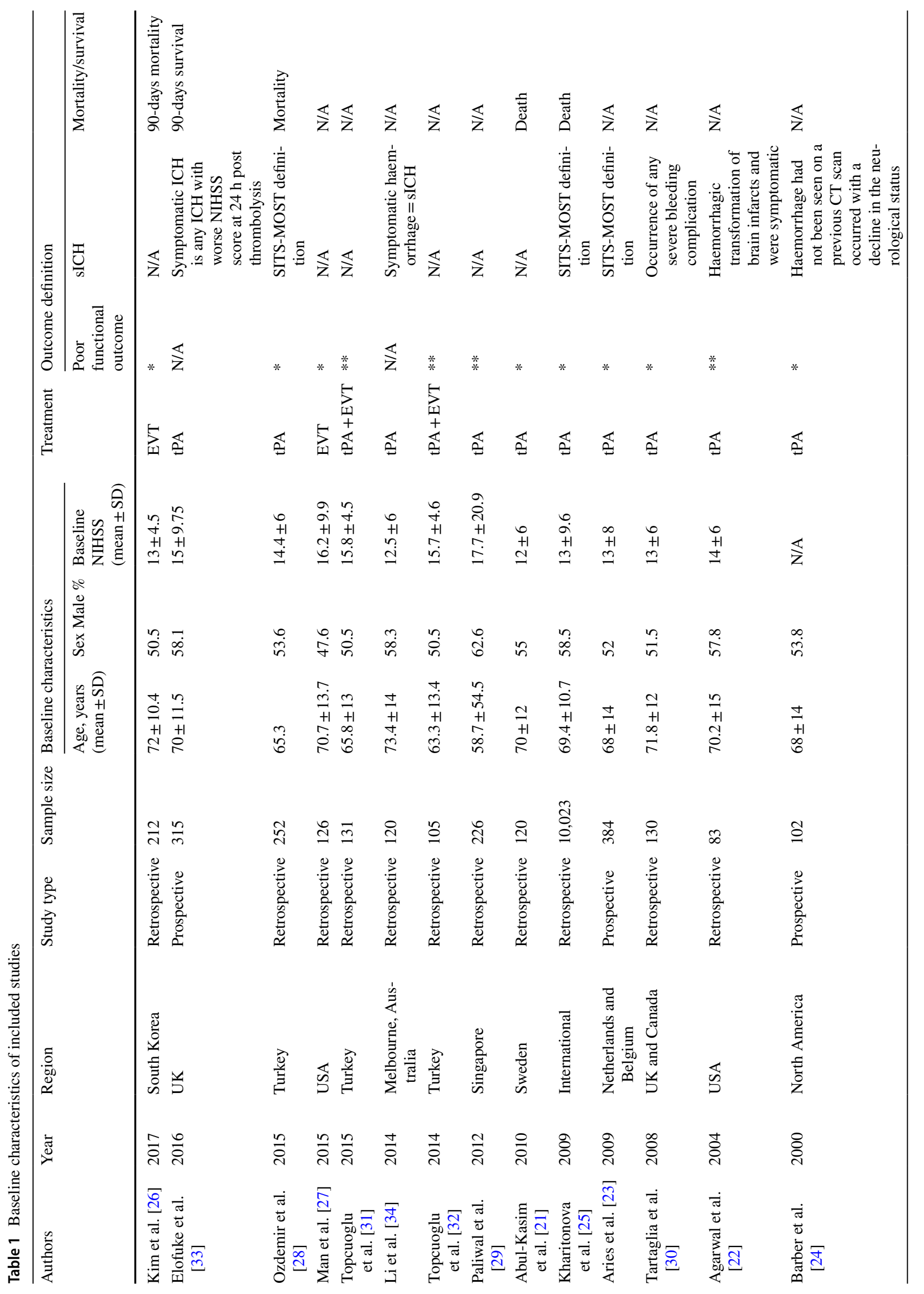




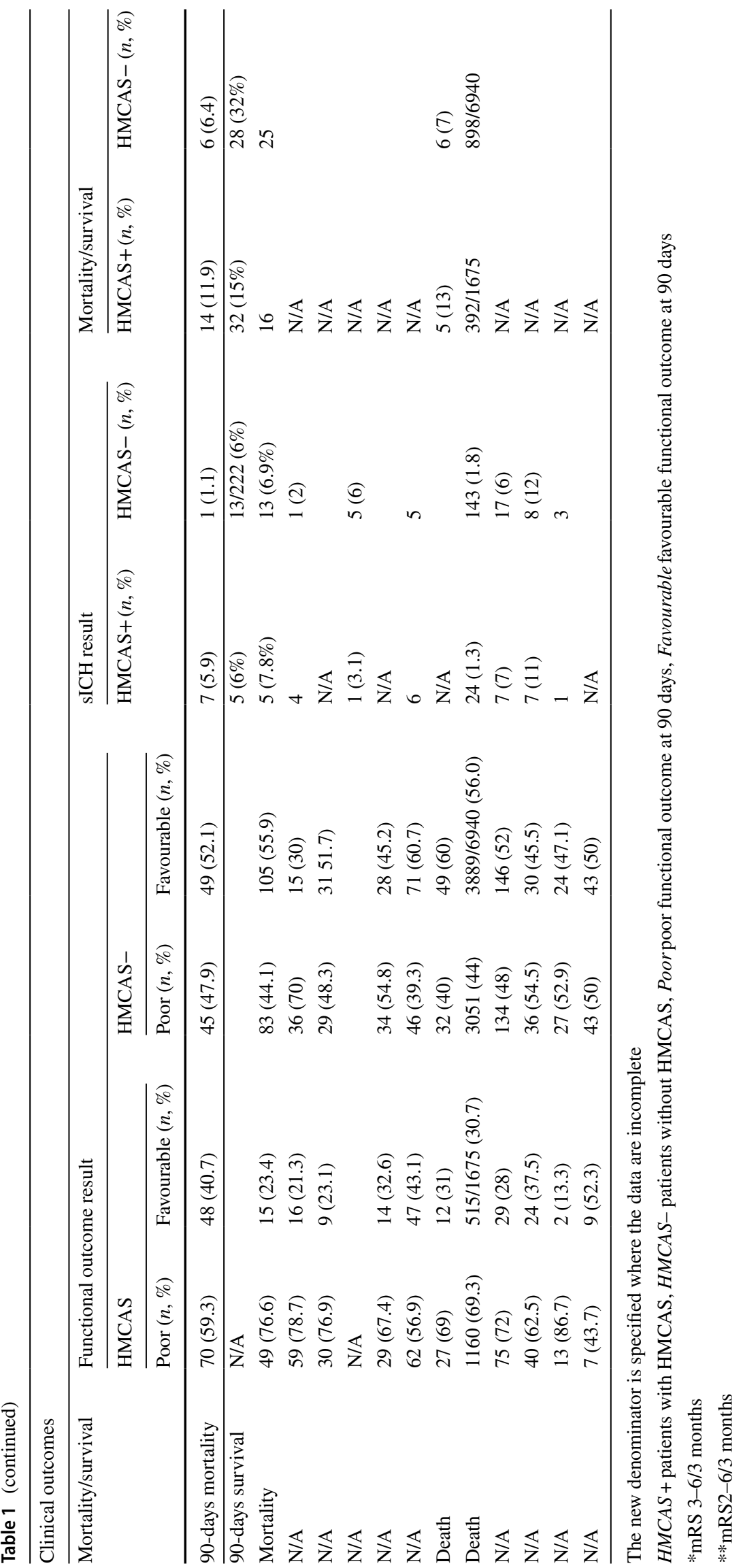




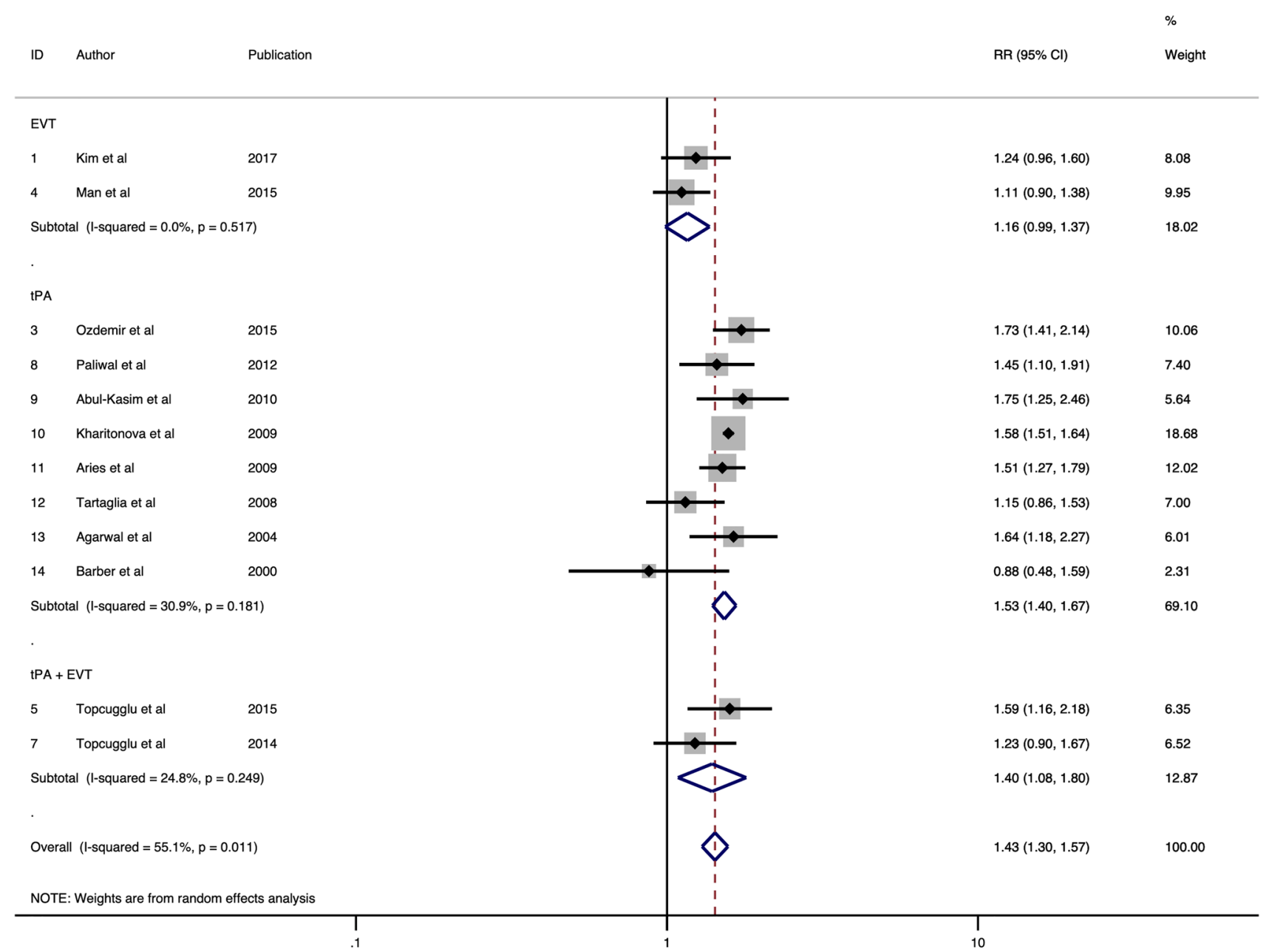

Fig. 2 Forest plot showing the association of the hyperdense middle cerebral artery sign with the risk of poor functional outcome in acute ischaemic stroke patients receiving thrombolysis or/and mechanical thrombectomy

association of HMCAS with mortality risk in EVT patients could not be done due to limited number of studies. The sensitivity and specificity stratified by treatment subgroups are provided in Table 2.

\section{Discussion}

This meta-analysis reports on the association of pre-treatment HMCAS with functional outcome, mortality and sICH in acute ischaemic stroke patients receiving reperfusion therapy. This meta-analysis revealed that HMCAS is associated with increased risk of poor functional outcome at 90 days, though its prognostic sensitivity is low. HMCAS is significantly associated with increased risk of poor functional outcomes at 90 days in patients treated with tPA or $\mathrm{EVT}+\mathrm{tPA}$, however the association failed to reach statistical significance in EVT subgroup. HMCAS was not significantly associated with risk of sICH or mortality at 90 days.
Interestingly, HMCAS was associated with increased sICH risk following EVT, though the association didn't reach statistical significance. The meta-analyses on HDMCA and mortality risk in EVT patients could not be done due to lack of sufficient number of studies. Further studies are required to study the association of HMCAS with mortality and $\mathrm{SICH}$ risks following EVT.

Our analyses showed that patients with HMCAS on pre-treatment NCCT was 1.43 more likely to have a poor functional outcome at 90 days as compared to patients without HMCAS. There was no significant difference of sICH between HMCAS positive and HMCAS negative patients (RR 0.91). There was no significant association between HMCAS and risk of mortality at 90 days (RR 1.34). Our findings on risk ratios are consistent with the results of a recent systematic review and meta-analysis on association of HMCAS with functional outcomes (RR 1.56; 95\% CI $1.50-1.62 ; p=0.25)$ and sICH (RR 0.97; 95\% CI $0.72-1.30 ; p=0.15$ ) [35]. However, the previous analyses 
Table 2 Sensitivity and specificity of HMCAS in predicting functional outcome, sICH and mortality

\begin{tabular}{|c|c|c|c|c|c|c|}
\hline \multirow[t]{2}{*}{ Treatment } & \multicolumn{2}{|c|}{ Poor functional outcome } & \multicolumn{2}{|l|}{$\mathrm{sICH}$} & \multicolumn{2}{|l|}{ Mortality } \\
\hline & tPA & Total & tPA & Total & $\mathrm{tPA}$ & Total \\
\hline $\begin{array}{l}\text { Interstudy variation in sensitivity } \\
\text { (ICC_SEN) }\end{array}$ & $0.07(0.00-0.14)$ & $0.08(0.01-0.15)$ & $0.10(0.00-0.22)$ & $0.21(0.02-0.40)$ & $0.04(0.00-0.11)$ & $0.05(0.00-0.13)$ \\
\hline $\begin{array}{l}\text { Interstudy variation in sensitivity } \\
\text { (MED_SEN) }\end{array}$ & $0.61(0.57-0.70)$ & $0.63(0.58-0.69)$ & $0.64(0.57-0.76)$ & $0.71(0.62-0.83)$ & $0.59(0.54-0.70)$ & $0.60(0.55-0.70)$ \\
\hline $\begin{array}{l}\text { Interstudy variation in specificity } \\
\text { (ICC_SPE) }\end{array}$ & $0.11(0.00-0.22)$ & $0.14(0.03-0.24)$ & $0.06(0.00-0.12)$ & $0.10(0.01-0.18)$ & $0.24(0.00-0.49)$ & $0.22(0.00-0.43)$ \\
\hline $\begin{array}{l}\text { Interstudy variation in specificity } \\
\text { (MED_SPE) }\end{array}$ & $0.65(0.59-0.74)$ & $0.67(0.61-0.75)$ & $0.61(0.56-0.68)$ & $0.64(0.59-0.71)$ & $0.72(0.62-0.88)$ & $0.71(0.62-0.85)$ \\
\hline AUROC & $0.61(0.57-0.65)$ & $0.60(0.56-0.64)$ & $0.53(0.48-0.57)$ & $0.56(0.52-0.61)$ & $0.45(0.40-0.49)$ & $0.47(0.43-0.52)$ \\
\hline Heterogeneity (Chi-square) & 138.749 & 331.478 & 42.649 & 88.019 & 124.699 & 162.541 \\
\hline Heterogeneity (I-square) & 99 (98-99) & $99(99-100)$ & $95(92-99)$ & $98(96-99)$ & $98(97-99)$ & 99 (98-99) \\
\hline Sensitivity & $0.38(0.30-0.47)$ & $0.43(0.35-0.51)$ & $0.29(0.19-0.41)$ & $0.39(0.24-0.56)$ & $0.39(0.29-0.49)$ & $0.42(0.32-0.53)$ \\
\hline Specificity & $0.81(0.72-0.87)$ & $0.75(0.66-0.82)$ & $0.70(0.62-0.76)$ & $0.65(0.56-0.73)$ & $0.65(0.40-0.83)$ & $0.61(0.40-0.79)$ \\
\hline Positive Likelihood Ratio & $1.9(1.6-2.4)$ & $1.7(1.5-2.1)$ & $0.9(0.7-1.2)$ & $1.1(0.8-1.4)$ & $1.1(0.7-1.7)$ & $1.1(0.8-1.5)$ \\
\hline Negative Likelihood Ratio & $0.77(0.72-0.82)$ & $0.76(0.71-0.80)$ & $1.02(0.92-1.14)$ & $0.94(0.79-1.13)$ & $0.95(0.76-1.18)$ & $0.95(0.78-1.15)$ \\
\hline Diagnostic Odds Ratio & $3(2-3)$ & $2(2-3)$ & $1(1-1)$ & $1(1-2)$ & $1(1-2)$ & $1(1-2)$ \\
\hline
\end{tabular}

Number of quadratics points is greater than number of observations for EVT and tPA + EVT treatment subgroups

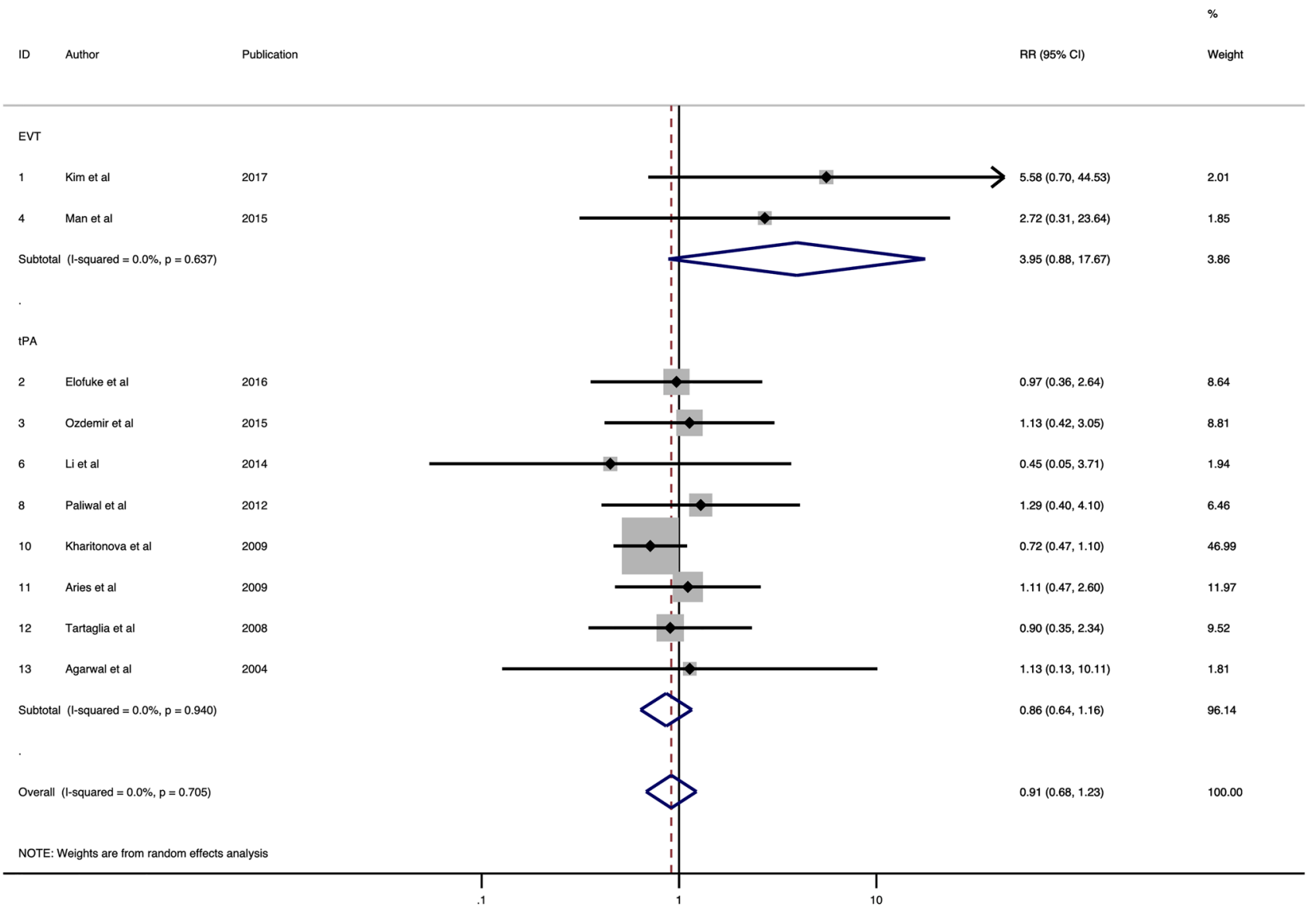

Fig. 3 Forest plot showing the association of the hyperdense middle cerebral artery sign with the risk of symptomatic intracerebral haemorrhage in acute ischaemic stroke patients receiving thrombolysis or/and mechanical thrombectomy 


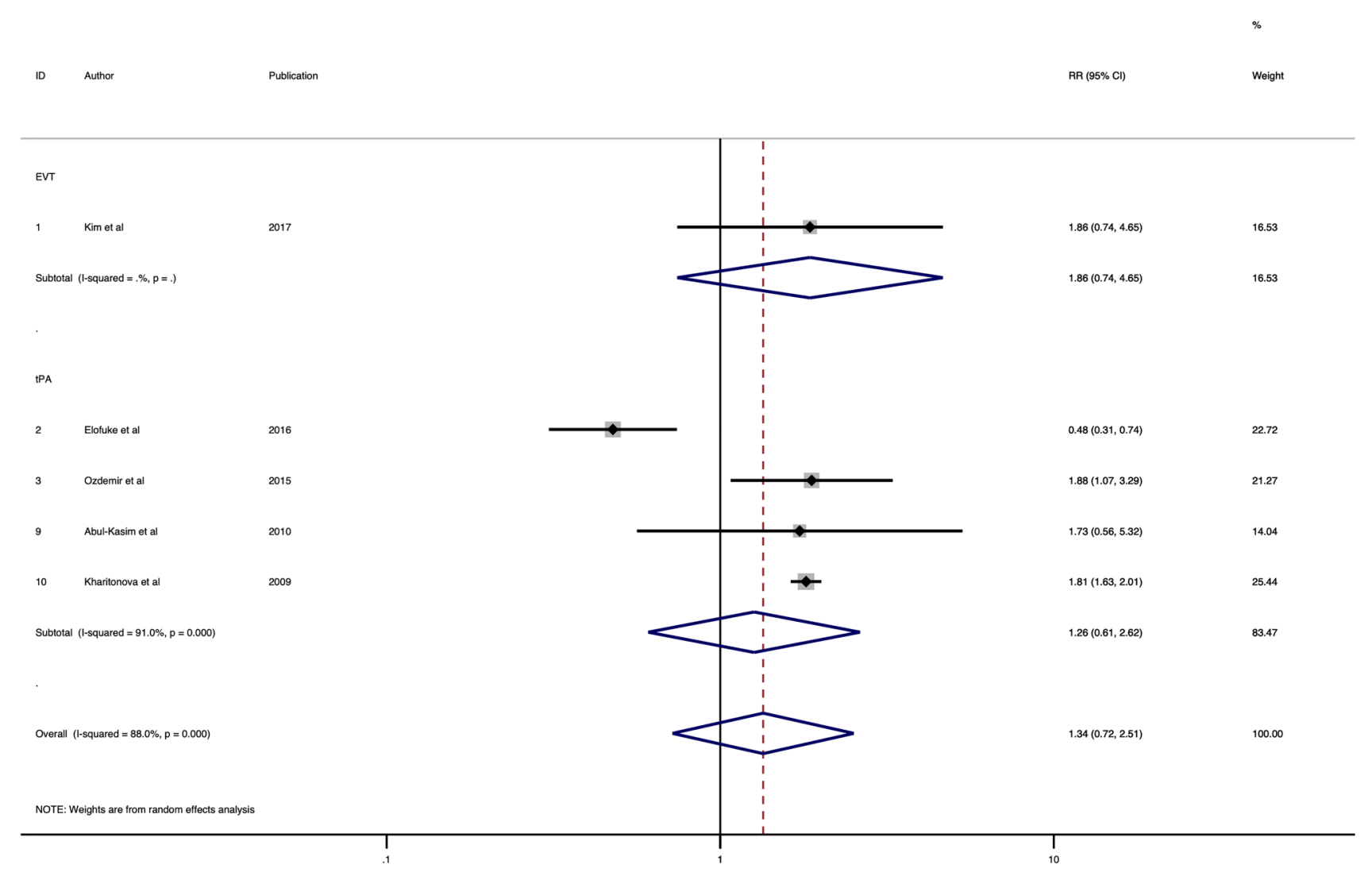

Fig. 4 Forest plot showing the association of the hyperdense middle cerebral artery sign with the risk of mortality in acute ischaemic stroke patients receiving reperfusion therapy

didn't stratify patients based on treatment type. Four studies on AIS patients receiving EVT and tPA were included in the current study [26, 27, 31,32]. We found that patients with HMCAS receiving EVT (RR 1.1) were associated with lower rates of poor functional outcomes at 90 days as compared to patients receiving tPA (RR 1.53) and combined therapies (RR 1.40).

Sensitivity and specificity analyses on the association of HMCAS with clinical outcomes are reported elsewhere (Table 2). Overall, the prognostic sensitivity and specify of HMCAS is poor, with sensitivity lower than the specificity. The sensitivity of HMCAS in predicting a poor functional outcome was 0.43 (95\% CI $0.35-0.51$ ). In AIS patients receiving tPA, the sensitivity was 0.38 (95\% CI 0.30-0.47). Similarly, the sensitivity of HMCAS in predicting sICH was 0.39 (95\% CI 0.24-0.56) and 0.29 (95\% CI 0.19-0.41) overall and in tPA subgroup, respectively. With regards to mortality, the sensitivity of HMCAS was 0.42 (95\% CI $0.32-0.53$ ) and 0.39 (95\% CI 0.29-0.49) overall and in tPA subgroup, respectively. These findings demonstrate that HMCAS is not a robust prognostic biomarker in AIS patients receiving reperfusion therapy.

Future research should be directed on the combined use of HMCAS with other baseline characteristics or biomarkers.
For example, other imaging biomarker considerations, such as arterial collateral status are important in determining the prognosis of AIS patient [36-39]. Although, HMCAS might not be the initial preference in predicting clinical outcome in the tertiary care settings, it may be relatively easy to appreciate in an emergency setting and could reduce the time and complexity of acute stroke workflow $[5,22]$. This suggests a potential prognostic use of HMCAS in low-resourced settings where only NCCT imaging is available. This is also applicable in telestroke services where a neurologist can provide remote reperfusion treatments to AIS patients, especially in the Coronavirus disease (COVID-19) settings when minimizing viral exposure to healthcare workers is of public health concern [40].

There are several limitations to this study. The heterogeneity in the patient population due to varying patients' selection criteria across several studies was one of the limitations. However, the use of random-effect modelling should mitigate the risk and limitations of heterogeneity in this metaanalysis. Secondly, the CT slice thickness was not uniform across all studies. Varying slice thickness on NCCT imaging affect the accuracy of HMCAS identification by the reporting radiologist. Thin-sliced NCCT is recommended to provide more sensitive and reliable detection of MCA clots [41, 42]. 
Fig. 5 Funnel plots showing studies on association of HMCAS with A functional outcome at 90 days, $\mathbf{B}$ sICH and C mortality

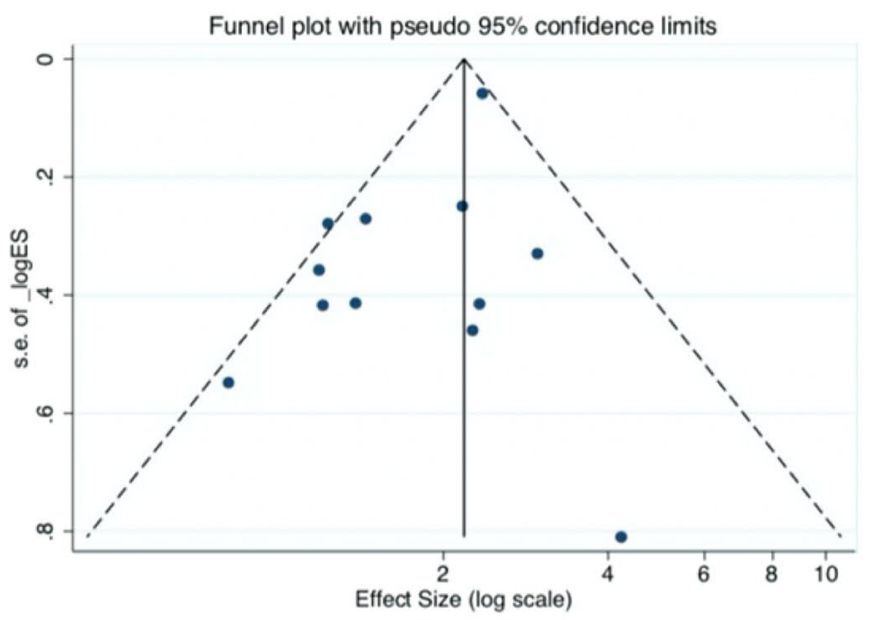

(A) Poor functional outcome at 90 days

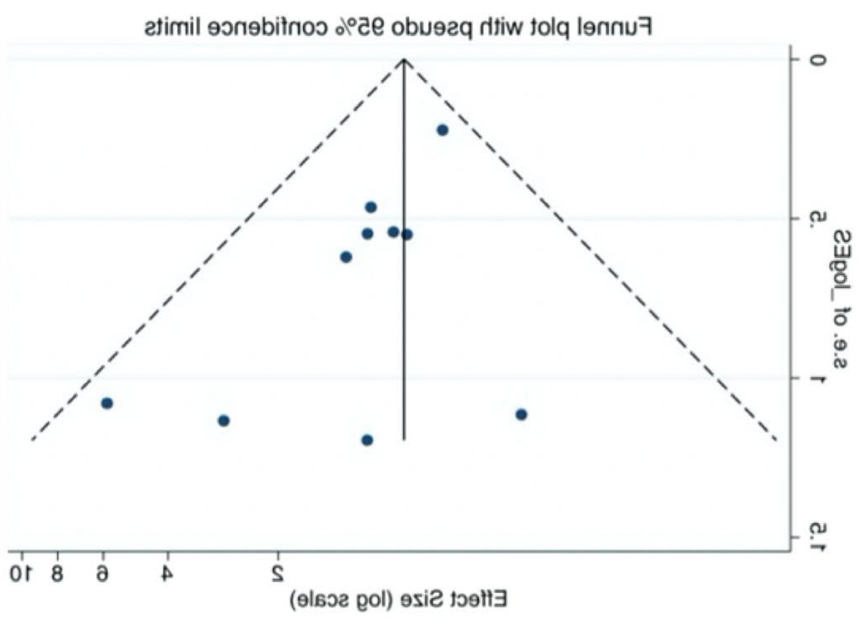

(B) Symptomatic intracerebral hemorrhage

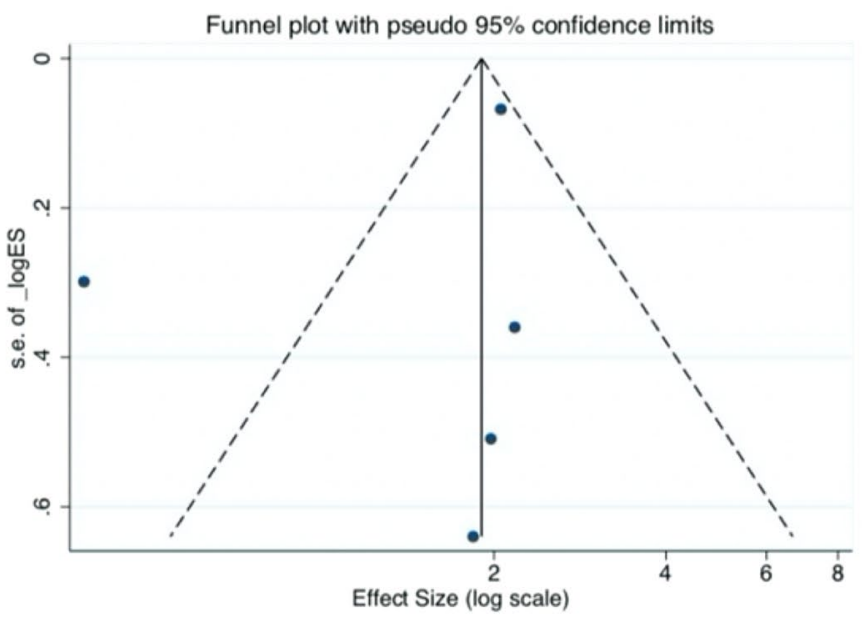

(C) Mortality 
Another limitation of this study was a relatively small sample size. There were only 4 articles identified with patients receiving EVT or combined therapies. Therefore, subgroup analysis for the association of HMCAS and mortality in EVT patients could not be performed. Short term clinical outcomes at $24 \mathrm{~h}$ was considered in this meta-analysis; however, short term outcomes were defined differently across studies, including early neurological deterioration [23], neurological improvement at $24 \mathrm{~h}$ [28], dramatic neurological improvement at $24 \mathrm{~h} \mathrm{[28],} \mathrm{rapid} \mathrm{neurological} \mathrm{improve-}$ ment [34] and early dramatic recovery [31, 32]. Overall, the HMCAS positive patients were more likely to be associated with increasing rates of early neurological deterioration [23] (39\% vs 28\%) and lower rates of neurological improvement $[28,31,32,34]$ as compared to HMCAS negative patients. Similarly, only 3 articles, with varying angiographic assessment criteria: mTICI [26, 27], TCD monitoring (TIBI) [28], were included to study the association of HMCAS with angiographic recanalization. This was not sufficient to conduct a meta-analysis. One study included 10,023 patients from the Safe Implementation of Thrombolysis in Stroke International Stroke Thrombolysis Register (SITS-ISTR) which would skew the results [25]. The risk ratio would deviate towards this study's result since it accounted for more than half of the cumulative weights in the meta-analysis. Furthermore, we did not report on the association of location (proximal vs distal) $[27,34,43]$ and attenuation (persistence vs disappearance) $[29,44]$ of HMCAS which may be of prognostic significance. It is suggested that proximal M1 and full-length $\mathrm{M} 1$ are more likely to have a lower rate of long-term favourable functional outcome [27, 43]. Paliwal et al. suggested that persistence of HMCAS can be an early prognostic factor of poor functional outcome (OR 3.352; 95\% CI 1.991-11.333; $p=0.039$ ) [29]. Kharitonova et al. reported that the disappearance of HMCAS was associated with twice the rates of good functional outcome relative to the persistent HMCAS [44]. Therefore, further studies are required to delineate the accurate relationship between HMCAS and clinical outcomes following AIS across various treatment subgroups. Moreover, the interaction of HMCAS with good and poor collaterals [39] and their role in mediating outcomes following reperfusion therapy is an another potential area of further research. Notably, the role of clot composition, including imaging features, such as clot density or clot perviousness, has been of increasing clinical interest as they may be useful in guiding treatment selection $[9,15]$. For example, it is postulated that a very dense artery, would be composed of RBC and thus amenable to aspiration with EVT; and less to IVT using alteplase for example [9]. The pathologic correlations of actual EVT-retrieved clot features with the preintervention non-invasive imaging features, such as HMCAS on NCCT or blooming artefact on MRI are also of pathophysiological significance [14, 45, 46].

\section{Conclusions}

This meta-analysis demonstrated that though HMCAS is associated with increased risk of poor functional outcomes, its sensitivity in predicting long-term functional outcome, mortality and sICH after reperfusion therapy is poor. Future studies to delineate the prognostic role of HMCAS in EVT patients are recommended. Moreover, the role of clot composition or clot density in guiding selection of optimal reperfusion therapy or EVT device warrant further research.

\section{Data availability statement}

The original contributions presented in the study are included in the article/Supplementary Information, further inquiries can be directed to the corresponding author.

Supplementary Information The online version contains supplementary material available at https://doi.org/10.1007/s13760-021-01720-3.

Acknowledgements We would like to acknowledge the support from the administrative staff and NSW wide partnering clinicians and investigators.

Author contributions SB conceived the study, contributed to the planning, draft and revision of the manuscript; supervision of the students. SB encouraged CS to investigate and supervised the findings of this work. CS and SB wrote the first draft of this paper. All authors contributed to the revision of the manuscript. All authors approved the final draft of the manuscript.

Funding Seed funding from the UNSW Medicine Mindgardens CAG funding 2017 for the Thrombolysis and Endovascular FLow Network (TEFLON) trial (Chief Investigator: SB) and funding for the NSW Brain Clot Bank (Chief Investigator: SB) from the NSW Ministry of Health (2019-2022) is acknowledged.

\section{Declarations}

Conflict of interest The authors declare that they have no conflict of interest.

Ethics approval All analyses were based on previously published studies; thus, no ethical approval and patient consent are required.

\section{References}

1. Birenbaum D, Bancroft LW, Felsberg GJ (2011) Imaging in acute stroke. West J Emerg Med 12(1):67-76

2. Gasparian GG, Sanossian N, Shiroishi MS, Liebeskind DS (2015) Imaging of occlusive thrombi in acute ischemic stroke. Int $\mathbf{J}$ Stroke 10(3):298-305. https://doi.org/10.1111/ijs.12435

3. Bastianello S, Pierallini A, Colonnese C, Brughitta G, Angeloni U, Antonelli M et al (1991) Hyperdense middle cerebral artery CT sign. Comparison with angiography in the acute phase of ischemic supratentorial infarction. Neuroradiology 33(3):207. https://doi. org/10.1007/BF00588219 
4. Koo CK, Teasdale E, Muir KW (2000) What constitutes a true hyperdense middle cerebral artery sign? Cerebrovasc Dis 10(6):419-423

5. Leys D, Pruvo JP, Godefroy O, Rondepierre P, Leclerc X (1992) Prevalence and significance of hyperdense middle cerebral artery in acute stroke. Stroke 23(3):317-324. https://doi.org/10.1161/01. str.23.3.317

6. Zou M, Churilov L, He A, Campbell B, Davis SM, Yan B (2013) Hyperdense middle cerebral artery sign is associated with increased risk of hemorrhagic transformation after intravenous thrombolysis for patients with acute ischaemic stroke. J Clin Neurosci 20(7):984-987. https://doi.org/10.1016/j.jocn.2012.10.013

7. Mair G, Boyd Elena V, Chappell Francesca M, von Kummer R, Lindley Richard I, Sandercock P et al (2015) Sensitivity and specificity of the hyperdense artery sign for arterial obstruction in acute ischemic stroke. Stroke 46(1):102-107. https://doi.org/ 10.1161/STROKEAHA.114.007036

8. Bhaskar S, Stanwell P, Cordato D, Attia J, Levi C (2018) Reperfusion therapy in acute ischemic stroke: dawn of a new era? BMC Neurol 18(1):8. https://doi.org/10.1186/s12883-017-1007-y

9. Luthman AS, Bouchez L, Botta D, Vargas MI, Machi P, Lövblad $\mathrm{K}-\mathrm{O}$ (2020) Imaging clot characteristics in stroke and its possible implication on treatment. Clin Neuroradiol 30(1):27-35. https:// doi.org/10.1007/s00062-019-00841-w

10. Santana Baskar P, Cordato D, Wardman D, Bhaskar S (2021) Inhospital acute stroke workflow in acute stroke-systems-based approaches. Acta Neurol Scand 143(2):111-120. https://doi.org/ 10.1111/ane. 13343

11. Bhaskar S, Stanwell P, Bivard A, Spratt N, Walker R, Kitsos GH et al (2017) The influence of initial stroke severity on mortality, overall functional outcome and in-hospital placement at 90 days following acute ischemic stroke: a tertiary hospital stroke register study. Neurol India 65(6):1252-1259. https://doi.org/10.4103/ 0028-3886.217947

12. Bhaskar S, Bivard A, Stanwell P, Attia JR, Parsons M, Nilsson M et al (2016) Association of cortical vein filling with clot location and clinical outcomes in acute ischaemic stroke patients. Sci Rep 6:38525. https://doi.org/10.1038/srep38525

13. Ravindran AV, Killingsworth MC, Bhaskar S (2021) Cerebral collaterals in acute ischaemia: implications for acute ischaemic stroke patients receiving reperfusion therapy. Eur J Neurosci 53(4):1238-1261. https://doi.org/10.1111/ejn.14955

14. Bhaskar S, Cordato D, Cappelen-Smith C, Cheung A, Ledingham D, Celermajer D et al (2017) Clarion call for histopathological clot analysis in "cryptogenic" ischemic stroke: implications for diagnosis and treatment. Ann Clin Transl Neurol 4(12):926-930. https://doi.org/10.1002/acn3.500

15. Bhaskar S, Saab J, Cappelen-Smith C, Killingsworth M, Wu XJ, Cheung A et al (2019) Clot histopathology in ischemic stroke with infective endocarditis. Can J Neurol Sci 46(3):331-336. https:// doi.org/10.1017/cjn.2019.8

16. Urrútia G, Bonfill X (2010) PRISMA declaration: a proposal to improve the publication of systematic reviews and meta-analyses. Med Clin (Barc) 135(11):507-511. https://doi.org/10.1016/j.medcli.2010.01.015

17. Murad MH, Sultan S, Haffar S, Bazerbachi F (2018) Methodological quality and synthesis of case series and case reports. BMJ Evid Based Med 23(2):60-63. https://doi.org/10.1136/ bmjebm-2017-110853

18. NHLBI (2014) Quality Assessment Tool for Observational Cohort and Cross-Sectional Studies. https://www.nhlbi.nih.gov/ health-pro/guidelines/in-develop/cardiovascular-risk-reduction/ tools/cohort. Accessed 4 Aug 2020

19. Faggion JCM, Atieh M, Zanicotti DG (2014) Reporting of sources of funding in systematic reviews in periodontology and implant dentistry. Br Dent J 216(3):109-112. https://doi.org/10. 1038/sj.bdj.2014.47

20. Wan X, Wang W, Liu J, Tong T (2014) Estimating the sample mean and standard deviation from the sample size, median, range and/or interquartile range. BMC Med Res Methodol 14(1):135. https://doi.org/10.1186/1471-2288-14-135

21. Abul-Kasim K, Brizzi M, Petersson J (2010) Hyperdense middle cerebral artery sign is an ominous prognostic marker despite optimal workflow. Acta Neurol Scand 122(2):132-139. https:// doi.org/10.1111/j.1600-0404.2009.01277.x

22. Agarwal P, Kumar S, Hariharan S, Eshkar N, Verro P, Cohen B et al (2004) Hyperdense middle cerebral artery sign: can it be used to select intra-arterial versus intravenous thrombolysis in acute ischemic stroke? Cerebrovasc Dis 17(2-3):182-190. https://doi.org/10.1159/000075789

23. Aries MJH, Uyttenboogaart M, Koopman K, Rödiger LA, Vroomen PC, De Keyser J et al (2009) Hyperdense middle cerebral artery sign and outcome after intravenous thrombolysis for acute ischemic stroke. J Neurol Sci 285(1):114-117. https://doi. org/10.1016/j.jns.2009.06.010

24. Barber PA, Demchuk AM, Zhang J, Buchan AM (2000) Validity and reliability of a quantitative computed tomography score in predicting outcome of hyperacute stroke before thrombolytic therapy. Lancet (Brit Edn) 355(9216):1670-1674. https://doi. org/10.1016/s0140-6736(00)02237-6

25. Kharitonova T, Ahmed N, Thorén M, Wardlaw JM, von Kummer R, Glahn J et al (2009) Hyperdense middle cerebral artery sign on admission CT scan-prognostic significance for ischaemic stroke patients treated with intravenous thrombolysis in the safe implementation of thrombolysis in Stroke International Stroke Thrombolysis Register. Cerebrovasc Dis 27(1):51-59. https://doi.org/10.1159/000172634

26. Kim SK, Baek BH, Lee YY, Yoon W (2017) Clinical implications of CT hyperdense artery sign in patients with acute middle cerebral artery occlusion in the era of modern mechanical thrombectomy. J Neurol 264(12):2450-2456. https://doi.org/10. 1007/s00415-017-8655-0

27. Man S, Hussain MS, Wisco D, Katzan IL, Aoki J, Tateishi $Y$ et al (2015) The location of pretreatment hyperdense middle cerebral artery sign predicts the outcome of intraarterial thrombectomy for acute stroke: hyperdense MCA sign location and intraarterial thrombectomy outcome. J Neuroimaging 25(2):263-268. https://doi.org/10.1111/jon.12115

28. Ozdemir O, Bas DF, Ozkan S, Colak E, Uzuner N (2015) A comparison of hyperdense internal carotid artery sign, hyperdense middle cerebral artery sign and middle cerebral artery dot sign in acute ischemic stroke. J Neurol Sci 32(4):636-648

29. Paliwal PR, Ahmad A, Shen L, Yeo LLL, Loh PK, Ng KWP et al (2012) Persistence of hyperdense middle cerebral artery sign on follow-up CT scan after intravenous thrombolysis is associated with poor outcome. Cerebrovasc Dis 33(5):446-452. https://doi.org/10.1159/000336863

30. Tartaglia MC, Di Legge S, Saposnik G, Jain V, Chan R, Bussière $\mathrm{M}$ et al (2008) Acute stroke with hyperdense middle cerebral artery sign benefits from IV rtPA. Can j neurol sci 35(5):583-587. https://doi.org/10.1017/S0317167100009367

31. Topcuoglu MAMD, Arsava EMMD, Akpinar EMD (2015) Clot characteristics on computed tomography and response to thrombolysis in acute middle cerebral artery stroke. J Stroke Cerebrovasc Dis 24(6):1363-1372. https://doi.org/10.1016/j.jstrokecer ebrovasdis.2015.02.017

32. Topcuoglu MAMD, Arsava EMMD, Kursun OMD, Akpinar EMD, Erbil BMD (2014) The utility of middle cerebral artery clot density and burden assessment by noncontrast computed tomography in acute ischemic stroke patients treated with thrombolysis. J 
Stroke Cerebrovasc Dis 23(2):e85-e91. https://doi.org/10.1016/j. jstrokecerebrovasdis.2013.08.026

33. Elofuke P, Reid JM, Rana A, Macleod MJ (2016) Disappearance of the hyperdense MCA sign after stroke thrombolysis: implications for prognosis and early patient selection for clot retrieval. J R Coll Physicians Edinb 46(2):81-86. https://doi.org/10.4997/ JRCPE.2016.203

34. Li Q, Davis S, Mitchell P, Dowling R, Yan B (2014) Proximal hyperdense middle cerebral artery sign predicts poor response to thrombolysis. PLoS ONE 9(5):e96123. https://doi.org/10.1371/ journal.pone.0096123

35. Sun H, Liu Y, Gong P, Zhang S, Zhou F, Zhou J (2019) Intravenous thrombolysis for ischemic stroke with hyperdense middle cerebral artery sign: a meta-analysis. Acta Neurol Scand 141(3):193-201. https://doi.org/10.1111/ane.13177

36. Bhaskar S, Bivard A, Stanwell P, Attia JR, Parsons M, Nilsson M et al (2016) Association of cortical vein filling with clot location and clinical outcomes in acute ischaemic stroke patients. Sci Rep 6(1):38525. https://doi.org/10.1038/srep38525

37. Bhaskar S, Bivard A, Stanwell P, Parsons M, Attia JR, Nilsson M et al (2017) Baseline collateral status and infarct topography in post-ischaemic perilesional hyperperfusion: an arterial spin labelling study. J Cereb Blood Flow Metab 37(3):1148-1162. https:// doi.org/10.1177/0271678X16653133

38. Bhaskar S, Bivard A, Parsons M, Nilsson M, Attia JR, Stanwell $P$ et al (2017) Delay of late-venous phase cortical vein filling in acute ischemic stroke patients: associations with collateral status. J Cereb Blood Flow Metab 37(2):671-682. https://doi.org/ $10.1177 / 0271678 \times 16637611$

39. Ravindran AV, Killingsworth MC, Bhaskar S (2020) Cerebral collaterals in acute ischemia: implications for acute ischemic stroke patients receiving reperfusion therapy. Eur J Neurosci. https://doi. org/10.1111/ejn.14955

40. Bhaskar S, Sinha A, Banach M, Mittoo S, Weissert R, Kass JS et al (2020) Cytokine storm in COVID-19-immunopathological mechanisms, clinical considerations, and therapeutic approaches: The REPROGRAM Consortium position paper. Front Immunol 11:1648. https://doi.org/10.3389/fimmu.2020.01648
41. Riedel CH, Zoubie J, Ulmer S, Gierthmuehlen J, Jansen O (2012) Thin-slice reconstructions of nonenhanced CT images allow for detection of thrombus in acute stroke. Stroke 43(9):2319-2323. https://doi.org/10.1161/strokeaha.112.649921

42. Heo JH, Kim K, Yoo J, Kim YD, Nam HS, Kim EY (2017) Computed tomography-based thrombus imaging for the prediction of recanalization after reperfusion therapy in stroke. J Stroke 19(1):40-49. https://doi.org/10.5853/jos.2016.01522

43. Çetiner M, Aydin HE, Güler M, Canbaz Kabay S, Zorlu Y (2018) Predictive factors for functional outcomes after intravenous thrombolytic therapy in acute ischemic stroke. Clin Appl Thromb Hemost 24(9_suppl):171S-S177. https://doi.org/10.1177/10760 29618796317

44. Kharitonova T, Thorén M, Ahmed N, Wardlaw JM, von Kummer $\mathrm{R}$, Thomassen L et al (2009) Disappearing hyperdense middle cerebral artery sign in ischaemic stroke patients treated with intravenous thrombolysis: clinical course and prognostic significance. J Neurol Neurosurg Psychiatry 80(3):273-278. https://doi.org/10. 1136/jnnp.2008.150185

45. Bhaskar S, Saab J, Cappelen-Smith C, Killingsworth M, Wu XJ, Cheung A et al (2019) Clot histopathology in ischemic stroke with infective endocarditis. Can J Neurol Sci/J Can Sci Neurol 46(3):331-336. https://doi.org/10.1017/cjn.2019.8

46. Liebeskind SD, Sanossian HN, Yong PW, Starkman LS, Tsang DM, Moya MA et al (2011) CT and MRI early vessel signs reflect clot composition in acute stroke. Stroke 42(5):1237-1243. https:// doi.org/10.1161/STROKEAHA.110.605576

Publisher's Note Springer Nature remains neutral with regard to jurisdictional claims in published maps and institutional affiliations. 